\title{
A Note on the Copy vs. Multidominance Theories of Movement*
}

\section{Luis Vicente}

Universität Potsdam. Institut für Linguistik

Karl Liebknechtstraße 24-25. 14776 Golm. Germany

vicente@ling.uni-potsdam.de

http://www.luisvicente.net

\begin{abstract}
This article considers two different aspects of A-bar movement, namely (a) an analysis of reconstruction effects in terms of deletion at LF, and (b) Johnson's (2007) decompositional analysis of quantifiers as a means to derive the correct interpretation of A-bar chains. By examining how these two proposals mesh with each other, it is possible to compare the copy theory of movement to a multidominance alternative. We will see that each approach to movement must make use of slightly different assumptions in order to derive the correct results, thus establishing a basis for a potential comparison between the two of them.
\end{abstract}

Key words: quantification, A-bar movement, copy theory, multidominance, syntax, semantics.

\section{Table of Contents}

\section{Introduction}

2. Reconstruction and copy deletion

\section{Quantifiers, variables,} and multidominance

4. A copy theoretic implementation and its consequences
5. A multidominance implementation and its consequences

6. General conclusions and outlook

References

This article was written while I was a postdoctoral research fellow at the University of California, Santa Cruz. I want to thank all the members of UCSC Linguistics for creating a wonderful environment in which to carry out this (and other) research. Specifically to this paper, many thanks are due to Robert Henderson, Jim McCloskey, Kyle Johnson, and an anonymous reviewer for valuable comments and discussion. I also want to thank Angel Gallego and Jordi Fortuny for their invitation to contribute to this volume. Financial support was provided by the department of Education and Research of the Basque Government (postdoctoral grant BFI07.32). All remaining errors and shortcomings are nobody's fault but mine. 


\section{Introduction}

Let us begin by considering the proper analysis of (1).

\section{(1) Randy read every book}

Something that we know about this example is that its surface syntax cannot be directly translated into a legitimate semantics. The problem lies on the fact that, while the verb requires its complement to be of type $\langle\mathrm{e}\rangle$, every book is of type $\langle$ ett $\rangle$. In the GB era, this problem was solved by moving the QP to a higher structural position, following the analysis of May (1977). Quantifier Raising is a case of Abar movement, and since traces of A-bar movement were taken to be equivalent to definite descriptions, this particular compositionality problem was circumvented.

The situation changed when, for good theoretical and empirical reasons, trace theory was replaced with the copy theory of movement. Under an unadulterated version of the copy theory, applying QR to (1) would result in (2).

(2)

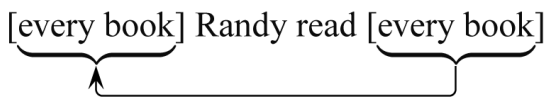

While this representation is faithful to the copy theory, it runs into the same problem as (1): the lower copy is still a generalized quantifier (even after movement takes place), and therefore it cannot sit in an object position. Fox $(2002,2003)$ has been quite influential in proposing that this issue be solved through a rule of Trace Conversion. This rule effectively turns the lower copy of a moved constituent into a definite description. Furthermore, it also inserts a variable that can be bound by the higher copy, ensuring that both copies are interpreted as members of the same movement chain. Consider, for example, the formulation in Johnson (2007:9), which is equivalent to the ones provided in Fox $(2002,2003)$ and Sauerland (2004).

(3) Trace Conversion

a. In the movement structure $\mathrm{DP}_{n}\left[{ }_{\phi} \ldots \mathrm{DP}_{n} \ldots\right]$, interpret $\phi$ as a function that maps $x$ to the meaning of $\phi[x / n]$.

b. The meaning of $\phi[x / n]$ is the result of replacing the head of every constituent with index $n$ in $\phi$ with the head the ${ }_{x}$, where $\llbracket$ the $\rrbracket=\lambda P$. $\llbracket$ the $\rrbracket[P \cap$ $\lambda y . y=x]$.

Given (3), (2) would be recast as (4). Note that the correct reading of this example is derived through binding of the variable introduced by clause (3b).

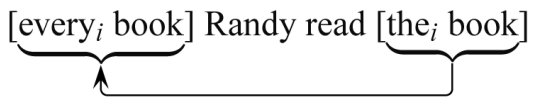

Trace Conversion does the job it is designed to do, but it does so in such a bruteforce way that it generates complaints. For instance, Johnson (2007:11) comments thus on it: 
"Fox's Trace Conversion has the undesirable property of letting a whole class of lexical items be ambiguous: in the cases we are examining, those lexical items are determiners. It resolves that ambiguity by syntactic rule. It claims that the meanings of determiners are not fixed, but change according to their position. It gives syntax the power to change lexical content. That's more than syntax should be allowed."

Johnson (2007) himself develops a way to replicate the results of Trace Conversion without actually literally transforming a quantifier into a definite-determiner-like variable. His proposal is framed into a multidominance analysis of movement, but this is not a logical necessity: as we shall see below, it is possible to recast his analysis into a copy-theoretic implementation. Importantly, these two modes to encode movement are not equivalent. We will see that each one must resort to slightly different assumptions to derive the correct syntax-to-PF and syntax-to-LF mappings. The article will achieve a null result - that is, the differences uncovered here have no tangible empirical consequences, at least with respect to the limited set of data that we explore. Nonetheless, the realization that such differences exist is, in and of itself, a valuable theoretical result: a number of previous studies on this topic (Kracht 2001, Starke 2001, Fitzpatrick and Groat 2005) had assumed that multidominance and the copy theory of movement were equivalent for all practical purposes. ${ }^{1}$ As a consequence, the discussion was reduced to conceptual and metatheoretical issues (general elegance, computational efficiency, economy of representation...). The differences discussed here make it possible to compare these two approaches in a more direct way.

The rest of the paper is organized as follows: in section 2, I introduce the empirical domain that the subsequent discussion will based on -namely, the semantics of wh- questions. In section 3, I introduce Johnson's (2007) reanalysis of Trace Conversion, which is based on a decompositional analysis of quantifiers. The rest of the paper explores how Johnson's analysis can be applied to the semantics introduced in section 2, focusing especially on the mapping from syntax to LF and PF. Section 4 does so assuming the copy theory as the way to represent movement chains, and section 5, assuming a multidominance alternative. Finally, section 6 concludes the exercise.

1. For instance, Frampton (2004:1) claims that "it has never been possible to establish empirical differences between [the copy theory] and [multidominance]". In fact, Frampton's paper is exceptional in that it does try to establish an empirical difference on the basis of Bulgarian multiple whfronting data. Also, as I was finishing this paper, I became aware of Sauerland (2007), who claims that a multidominance structure is necessary to properly analyze the indicated reading of (i).

(i) When I whistle, you say I shouldn't $\Delta_{1}$, but when I sing, you don't $\Delta_{2}$.

$\Delta_{1}=$ whistle $\quad \Delta_{2}=$ say I shouldn't sing

For reasons of space, I won't comment on Frampton's and Sauerland's papers, other than noting that, if their arguments are indeed correct, they constitute strong evidence in favour of multidominance approaches to movement. Note that this conclusion does not affect the argumentation in this paper, as I'll simply be defining a way in which the two approaches to movement can be further compared. 


\section{Reconstruction and copy deletion}

\subsection{Notes on the semantics of questions}

The argumentation in this paper is based on the analysis of wh- questions, so it is appropriate to begin by discussing some aspects of their grammar. Following the insight of Baker (1970), it has become customary to assume that wh- words contain an interrogative morpheme Q, which happens to be silent in English, but which receives a phonological realization in other languages (e.g., Japanese - $k a$ ). I will adopt the view in Reinhart (1997) and especially Hagstrom (1998) that Q is an existential quantifier over choice function variables. Also after Hagstrom, I take $\mathrm{Q}$ to carry all the quantificational force, the wh- word itself being non-quantificational (see Cable 2007 for a more extensive elaboration on this point). To illustrate Hagstrom's analysis, consider the following Japanese example.

$$
\begin{aligned}
& \text { [IP Randy-ga [DP nani-o } \quad t] \text { kaimasita] ka } \\
& \text { Randy.NOM what.ACC bought Q }
\end{aligned}
$$

"What did Randy buy?"

As illustrated above, Hagstrom starts his semantic analysis from the assumption that the Q morpheme - $k a$ starts as a sister of the wh- word nani-, and then moves to a position where it scopes over the whole clause. Evidence for this hypothesis comes primarily from locality effects (Hagstrom 1998:40-63), but also from the semantic parallelism between questions and indefinites (Hagstrom 1998:129-140). As already mentioned, he takes $-k a$ to be a quantifier. The consequence of the latter assumption is that the combination of -ka with the wh- word nani-forms a generalized quantifier (type $\langle\mathrm{ett}\rangle$ ) which cannot sit in an object position. This is the same problem posed by every book in (1). Hagstrom proposes to solve the type mismatch by (i) moving $-k a$ and (ii) transforming the trace of $-k a$ into a definite description -thus predating the introduction of Trace Conversion in Fox's work. More specifically, he turns this trace into a choice function variable, which is defined as a function $f$ that takes a set $P$ as its argument and outputs one member of that set. Since this element is essentially a determiner, I will refer to it as D.

$$
\llbracket \mathrm{D} \rrbracket=\lambda P . f(P)
$$

[choice function variable]

In order to bind this variable, the $\mathrm{Q}$ morpheme $-k a$ has to be a quantifier over choice functions -and, specifically, an existential quantifier (see Hagstrom 1998:131135 for justification). Thus, $\mathrm{Q}$ is defined as an element that takes an unsaturated proposition $G$ containing an undefined choice function and existentially binds it, creating a function from propositions to truth values. 
The attentive reader might have noticed that this is not enough to capture the semantics of a wh- question. The lexical entry in (7) is a function that creates a proposition. However, questions are not propositions, but sets of propositions. This additional aspect of meaning is contributed by the interrogative $\mathrm{C}^{0}$ head, which takes an unsaturated proposition requiring a choice function $(q)$ and returns the set of unsaturated propositions that can be saturated by the assignment of different choice functions $(p)$. This can be done by abstracting over $g$, the term representing the set of choice functions.

$$
\llbracket \mathrm{C}_{\text {interrogative }} \rrbracket=\lambda p \cdot \lambda q \cdot \lambda g \cdot[p=q(g)]
$$

With all these pieces in place, we can derive the logical form given in (9) below. ${ }^{2}$ For ease of exposition, I use English words instead of Japanese ones and omit irrelevant functional projections.

An $L F$ representation for (5)

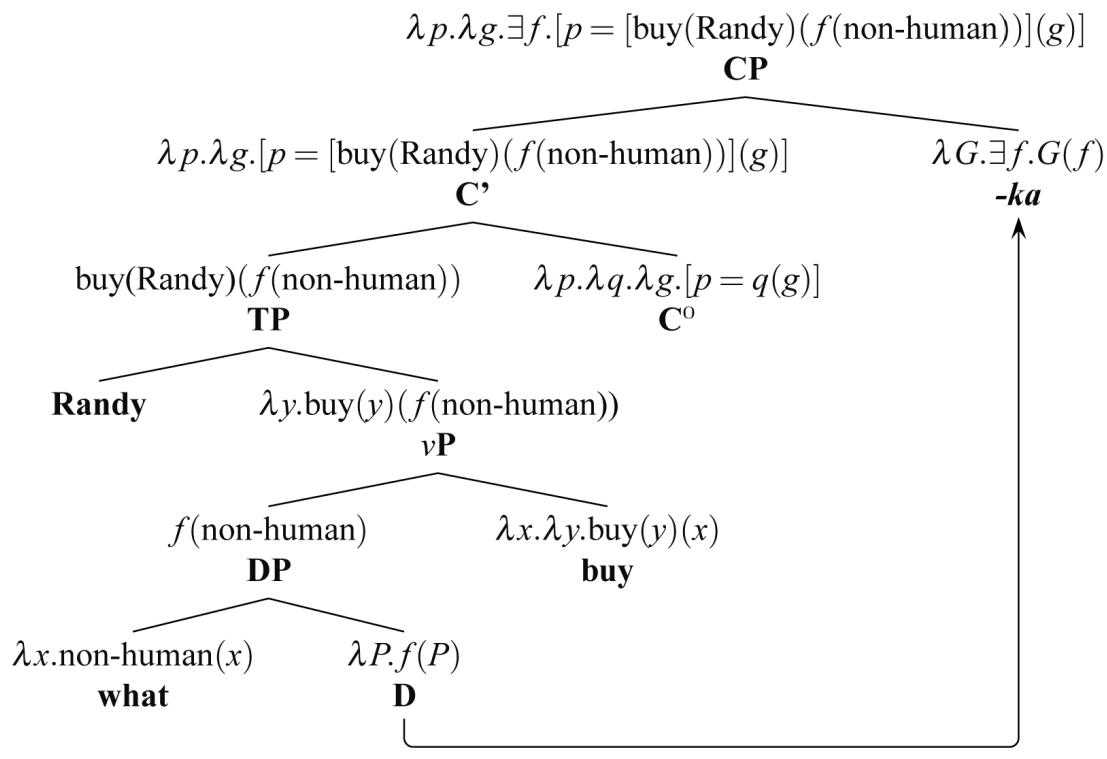

2. Hagstrom actually argues that $-k a$ undergoes long head movement into $\mathrm{C}^{0}$, creating a constituent with the latter. The reason behind this analysis is that he takes $-k a$ to be a head, which, given X-bar theory, can only move into an $\mathrm{X}^{0}$ position. Because of this, he defines $\mathrm{C}^{0}$ as an element that takes $-k a$ as an argument and returns a function from unsaturated propositions to existentially bound sets of propositions. Nonetheless, more recent theoretical developments (Toyoshima 2001, Vicente 2007,2009 ) allow for the type of head movement depicted in (9). The reason for adopting the latter option is that later on I will consider cases in English in which Q pied-pipes its nominal complement, rather than moving on its own. Without the modification, these cases would result in an implausible type of movement, namely, XP movement into a head position. 


\subsection{Reconstruction and copy deletion}

Hagstrom's (1998) analysis is based on Japanese and Sinhala data, and it is therefore designed to deal with the syntax and semantics of wh- in situ languages. The obvious question is whether it can be extended to cover wh- fronting languages (and if so, how the extension should proceed). Cable (2007) has an interesting take on this issue. He proposes that (9) is the correct representation of the semantics of wh- questions universally, irrespective of what the surface syntax of a particular language might look like. That is, even though English syntax forces movement of the entire wh- phrase, at LF it is as though only the silent Q morpheme had moved. Although this hypothesis might look a bit contrived at first sight, it automatically derives a pervasive property of wh- movement -namely, the fact that the restrictor of Q obligatorily reconstructs (Sauerland 1998, Takahashi 2006, amongst many others). The usual illustration of this effect is (10): in order to capture the Condition $\mathrm{C}$ violation, we must assume that a constituent containing Randy is necessarily interpreted under the scope of the coindexed pronoun.

$(10) *\left[\right.$ Which picture of Randy $\left._{i}\right]$ did he $_{i}$ throw away $t$ ?

If, as Cable proposes, only the silent Q morpheme is interpreted at LF in the scope position, it follows that the wh- phrase must be interpreted in a lower position. Thus, syntax produces, via Trace Conversion, the representation in (11a), which is then translated into the very simplified LF in (11b). ${ }^{3}$

(11) a. [which picture of Randy] did he throw away [the picture of Randy]

b. $\left[\mathrm{Q}_{i}\right]$ he threw away $\left[f_{i}\right.$ (picture of Randy)]

Reconstruction for anaphoric and variable binding illustrates the same point, though in a slightly less compelling way: if we considered (12a) and (12b) in isolation, we could well say that reconstruction is optional, and happens only if necessary to bring an anaphor/variable within the domain of its binder. However, (10) shows that reconstruction must happen nonetheless, even if it results in an ungrammatical sentence.

(12) a. [Which picture of himself ${ }_{i}$ ] did Randy ${ }_{i}$ throw away $t$ ?

b. [Which picture of himself ${ }_{i}$ ] did every ${ }_{i}$ man throw away $t$ ?

3. Note that this is not the only way in which obligatory reconstruction can potentially be captured: Takahashi (2006) and Hulsey and Takahashi (2009) propose a link between reconstruction and Case assignment. More precisely, picture of Randy must be interpreted at the foot of the chain because that is where it receives Case. Although the differences between this analysis and the one outlined in the main text make for an intriguing object of study, the choice between one or the other is inconsequential for the purposes of this paper. For one, in both analyses one begins with the syntactic representation in (11a), ends up with the LF in (11b), and must assume some process that derives the latter from the former - arguably, the same process in both analyses. 
Consider now what is required to get from the syntax in (11a) to the LF in (11b). What we need is a mechanism that eliminates some of the copies generated in narrow syntax and leaves just those required for a well-formed LF. A mechanism of this sort is assumed in much work on the syntax-PF interface-see, amongst others, Bobaljik (2002), Fanselow and Cavar (2002), Nunes (2004) Corver and Nunes (2006), Landau (2006), and references therein. Specifically, Landau (2006) proposes the following condition governing the phonetic realization of chains.

\section{(13) Economy of pronunciation}

Delete all chain copies at PF up to P-recoverability.

With this condition (or some equivalent) in place, we can ensure that PF will spell out only those links that are necessary to satisfy morpho-phonological well-formedness conditions. In the default case, the number of links spelled out reduces to one, as that is enough to recover the content of the constituent. Thus, the syntactic structure in (13) translates into the following PF representation, with deleted copies rendered in a light gray shade.

(14) [Which picture of Randy] did he throw away [the picture of Randy]

In order to deal with multiple copies at LF, we can augment (13) to read as in (15), which effectively allows us to perform the same process of copy deletion at LF. Moreover, I will also adopt (16) so that the processes of copy deletion at PF and LF don't influence each other. Interested readers are referred to Bobaljik (2002) for a study of the interactions of independent operations of copy deletion at both interfaces.

\section{(15) Economy of pronunciation and interpretation}

Delete all chain copies at PF up to P-recoverability, and at LF up to L-recoverability.

\section{(16) Modular Chain Resolution (Bobaljik 2002)}

The decision which chain link to pronounce or interpret is locally determined at PF or LF, respectively.

These principles apply to the structure in (11a) to derive the LF in (11b), reproduced in full in (17) below. After copy deletion applies, the chain in (11b)/(17b) still contains (i) a choice function quantifier, (ii) a choice function variable bound by the quantifier, and (iii) the wh- phrase, which provides the set inputted to the choice function (again, deleted copies appear in a light gray shade). These are the elements necessary to form a well-formed chain, and no superfluous constituents are left over. The fact that it is the lower copy of the wh- phrase that gets interpreted can be reduced to the lexical entries of the quantifier (7) and the variable (6) -i.e., the argument of the quantifier is already supplied by the verbal predicate, so there is no way the wh- phrase can compose with it. Rather, the wh- phrase must 
compose with the variable in order to supply the argument of the latter. In conclusion, the LF part of (15) is satisfied, and a legitimate logical form derived.

(17) a. [which picture of Randy] did he throw away [the picture of Randy]

b. $\left[\mathrm{Q}_{i}\right.$ picture of Randy] he threw away $\left[f_{i}\right.$ (picture of Randy)]

\section{Quantifiers, variables, and multidominance}

We have just seen that Hagstrom (1998) and Cable (2007) offer an analysis of whquestions that accounts for their major semantic properties in a rather elegant way. Of interest for the purposes of this article is the fact that their analyses are based on an application of Trace Conversion to the lower copy of the wh- phrase. That, we would like to improve. Now, bear in mind that Trace Conversion is a consequence of the fact that the "trace" of a quantifier must be a definite description, if semantic interpretation is to succeed. This conclusion is, as far as I can see, theoretically inescapable, and therefore the pertinent question is how it is to be implemented. Fox $(2002,2003)$ and Sauerland (2004) have proposed that the lower copy is literally transformed from a generalized quantifier into a definite description. More recently, though, Johnson (2007b) has developed an alternative analysis in which the definite description bound by the quantifier is there from the very beginning, without any actual conversion taking place. Johnson's approach is based on the hypothesis that quantifiers can be decomposed into a real quantificational part plus a definite-article-like variable. ${ }^{4}$ Crucially, though, Johnson does not merge both parts together. Rather, he merges the quantificational part at the scope position and the variable at the argument position. Then, a single NP restrictor is connected to both parts of the quantifier by multidominance. Under Johnson's analysis, an example like (5), repeated below as (18), would produce the syntactic representation in (19). ${ }^{5}$ We will consider the corresponding LF in a moment.

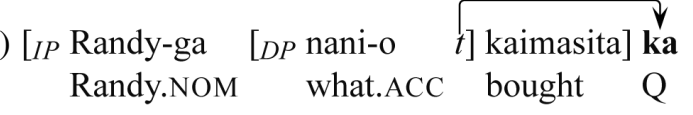

"What did Randy buy?"

4. In support of this proposal, Johnson cites Matthewson's (2001) observation that St'at'imcets Salish quantifiers embed a whole DP, rather than just a bare NP. Later on, Giannakidou (2004) and Etxeberria (2005) have argued at length that the Salish determiners are quantifier domain restrictors. Johnson is not explicit about whether he intends his determiner-like-variable to be a domain restrictor or not. This issue, however, is orthogonal to the discussion here. As discussed in the text, the important part is that the "trace" of the moved quantifier must be a definite description, lest semantic interpretation fails, and this much is captured by Johnson's proposal. Whether the "trace" is headed by an actual quantifier domain restrictor or some other type of variable is something that I defer to future research.

5. In actuality, Johnson illustrates his analysis with universal quantification. Nonetheless, his theory is designed to accomodate all types of quantification, so there is no danger in switching to whquestions here. 
(19) A multidominance syntax for (18)

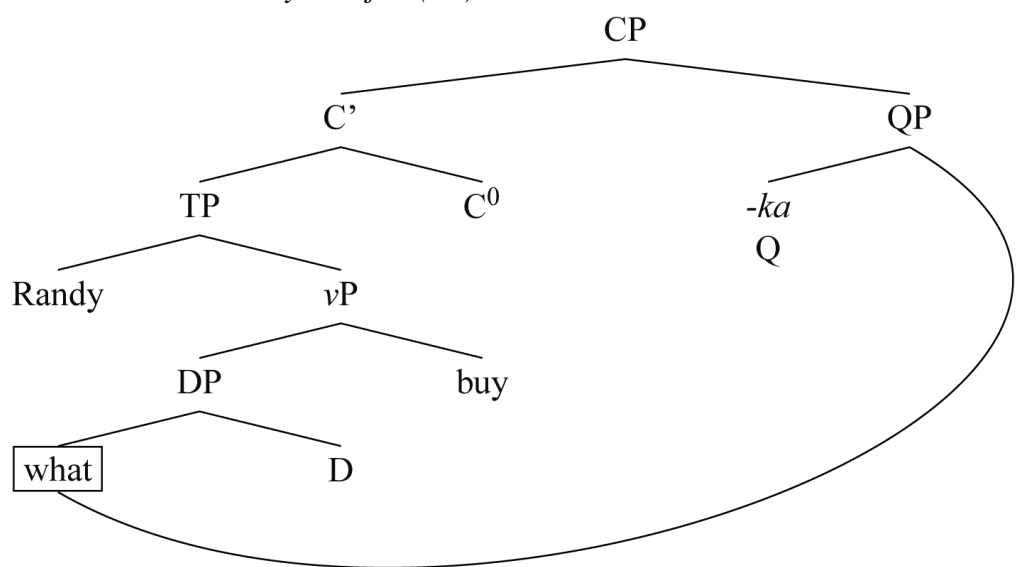

Johnson accomplishes a number of things with this structure. First of all (and most important for our purposes), by decomposing a quantifier into two separate parts, he avoids the troublesome process of transforming the lower copy from a generalized quantifier into a definite-description-like variable. Second, by merging the two parts of the quantifier in different parts of the tree, he can derive the correct quantificational structure. Finally, by resorting to multidominance as a way to link the two parts (as opposed to, e.g., binding), he can capture the locality effects inherent to wh- movement. Nonetheless, given the discussion in section 2.1, (19) is not directly translatable into a well-formed LF. The problem is that, after Hagstrom, we have defined Q as taking one single argument -i.e., the set of unsaturated propositions that obtains when $\mathrm{C}^{0}$ composes with TP. However, in (19), Q takes two arguments (i.e., the set of propositions plus nani- 'what'), which again takes us into a compositionality problem (20). Quite crucially, we cannot circumvent this problem simply by redefining $\mathrm{Q}$ as taking two arguments: that would lead to a situation where the NP restrictor is simultaneously interpreted at the argument and scope positions, thus failing to capture the reconstruction effects discussed in section 2.2. 
(20) A multidominance LF for (19)

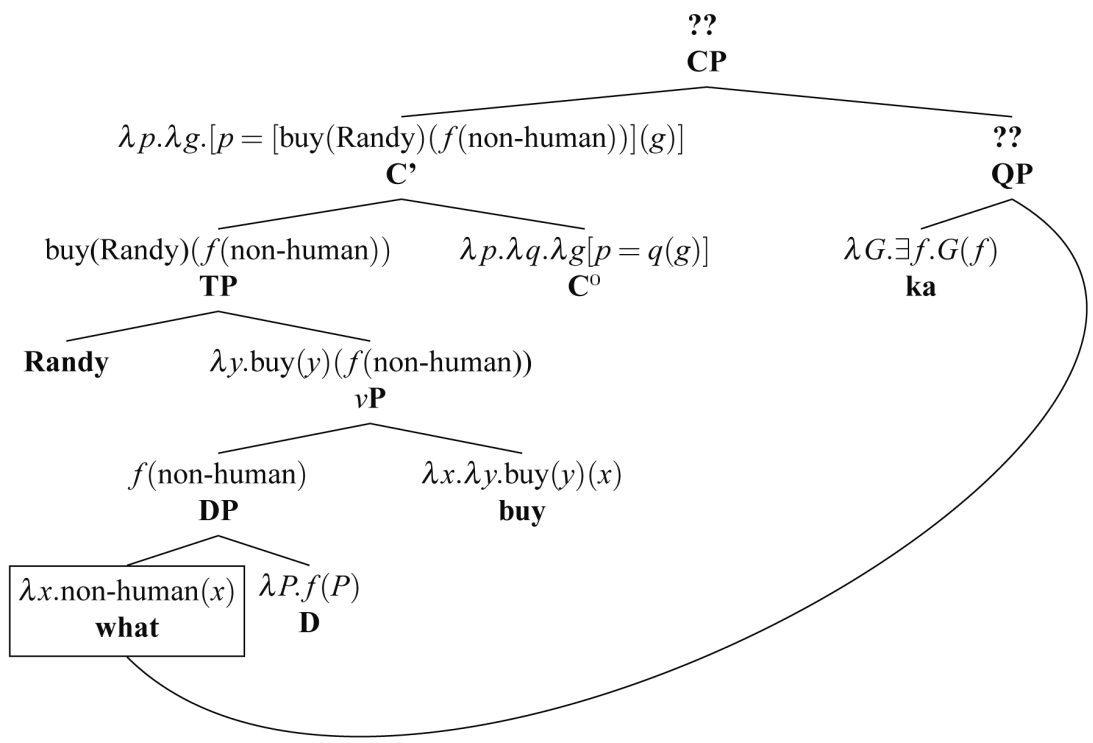

We don't want to reject Johnson's analysis as a whole, given that it provides a very elegant way to analyze Trace Conversion effects. We simply want to amend it so that we can still get this benefit without running into a compositionality problem. It is important to note, though, that Johnson's proposal has two independent ingredients: a decompositional analysis of quantifiers and a multidominance analysis of movement, only the former of which is crucial. As we shall see below, the amendments can be implemented both under a copy-theoretic and a multidominance approach to movement, and this is what offers us the possibility of comparing the two approaches directly. Due to certain difficulties inherent to the multidominance approach, it is easier to consider the copy-theoretic implementation first (4). With the conclusions reached there in place, we will then consider the multidominance implementation (5).

\section{A copy theoretic implementation and its consequences}

What I am going to argue for in this section is a way to implement Johnson's reformulation of Trace Conversion with the syntax and semantics of questions in whfronting languages (section 2.2). Therefore, let us assume that quantifiers can be divided into a quantificational and a variable part, allowing for the fact that the exact semantics of these two parts might vary depending on which quantifier we are dealing with. Thus, the $\mathrm{Q}$ morpheme breaks up into the two lexical entries in (6) and (7), repeated below as (21) and (22), respectively. 
Where I depart from Johnson is in the assumption that the quantificational and the variable part are merged in separate parts of the tree. I propose that, instead, they are merged together as part of the extended functional nominal structure, as illustrated below.

\section{A decomposed structure for generalized quantifiers}

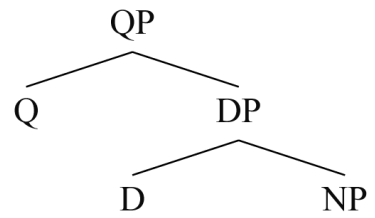

This structure is the only possibility if we do not admit multidominance: should we choose to merge $\mathrm{Q}$ directly in the scope position and then move NP from the argument position, we would have to perform an illicit movement operation namely, movement into a complement position.

\section{Illegal movement into the complement position of $Q$}

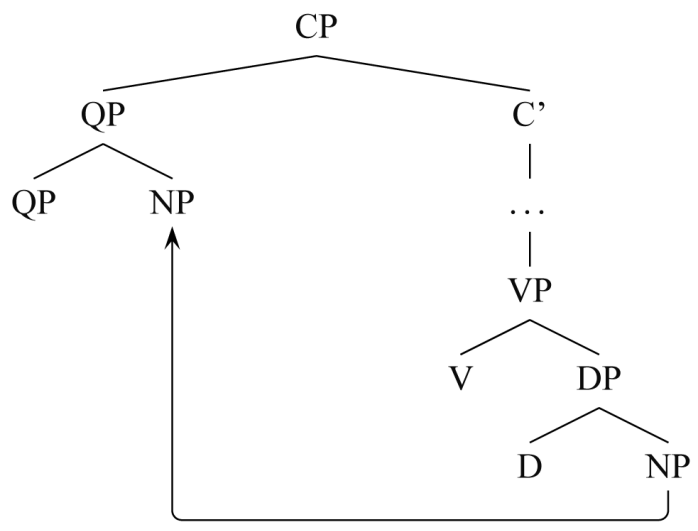

Thus, we can assume the syntactic structure in (26) below for the English whquestion (10), repeated here as (25). Note that so far we are replicating Cable's (2007) conclusion that wh- movement in English is movement of Q pied-piping its DP complement. Note also that one feature of this tree is the absence of any semantic information in all nodes. Given the Distributed Morphology claim that no phonological information is available during the narrow syntactic derivation, it seems reasonable to assume that no semantic information should be available either. 
(26) Syntactic structure for (25)

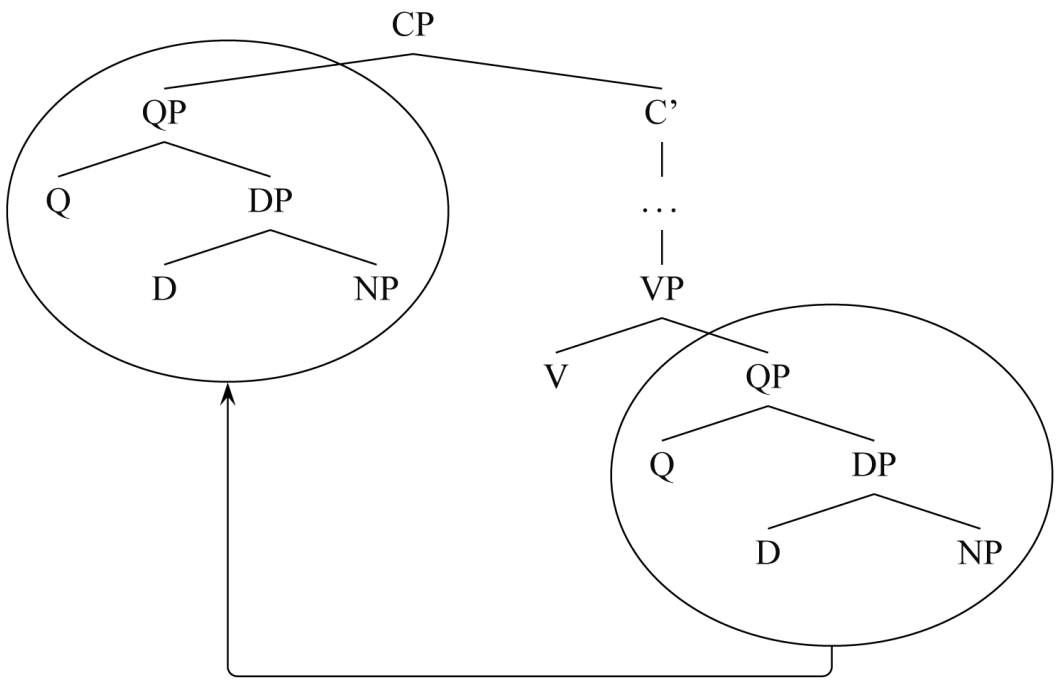

When this structure is transferred to LF, the economy principle in (13) applies so that only the copies necessary for a legitimate LF survive. This means, first of all, deletion of DP in the upper copy of movement, as already discussed in section 2.2 above. However, and very crucially for our purposes, it also means deletion of Q in the lower copy (see Cable 2007:176 for additional discussion). Let us see why: given (13), only one of the two copies of Q should be allowed at LF, since that is all that is needed to bind $\mathrm{D}$ and create the semantics of a movement chain. The upper copy of $\mathrm{Q}$ is necessary to establish the scope of the question. Therefore, it is the lower one that is deleted. The resulting structure is given below. ${ }^{6}$

6. Note that we must assume that, when Q is deleted in the argument position, the lower QP node becomes "transparent" for the computation of LF, so that DP and V can compose directly. This seems reasonable, since once Q is deleted from the argument position, the lower QP node can be computed through a vacuous instance of Functional Application, which passes up the denotation of DP unaltered. This remark also holds for comparable multidominance cases that we will consider in section 5 . 
(27) Logical Form for (25)

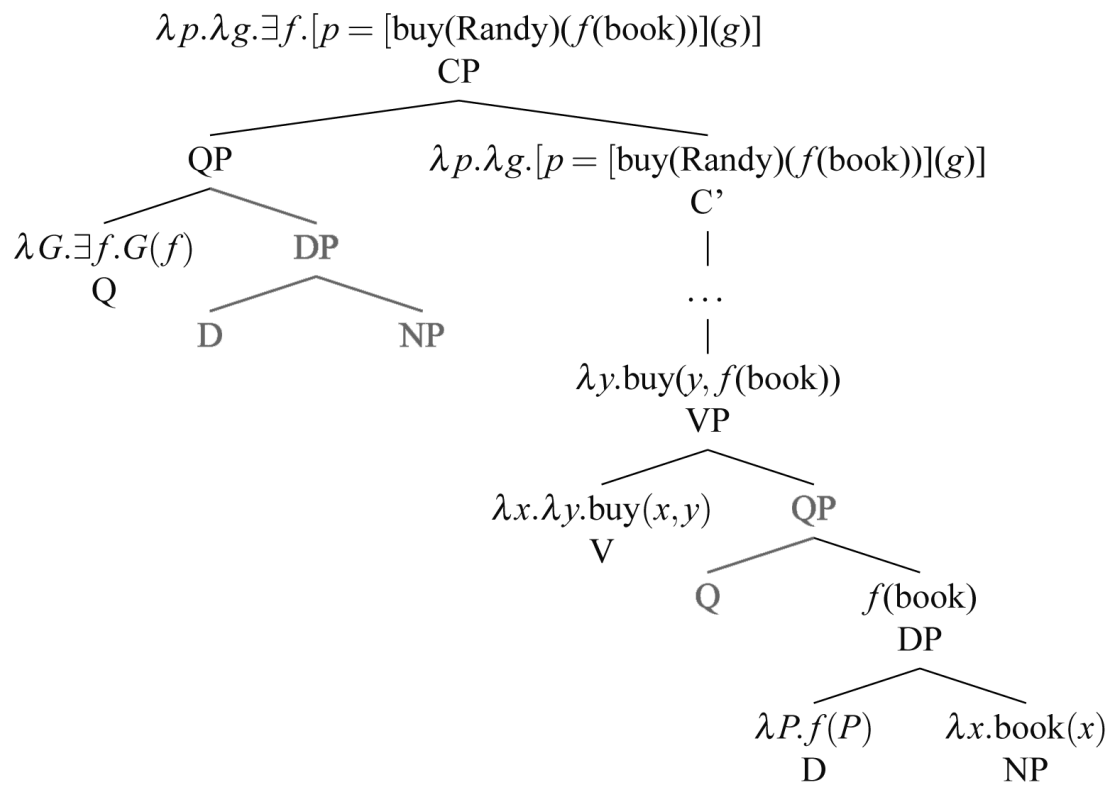

As we can see, (27) is formally identical to the logical form for questions discussed in section 2.1. Assuming Johnson's decompositional analysis of quantifiers and the independently motivated mechanism of copy deletion, we have been able to derive it without invoking a literal Trace Conversion process. I will assume, therefore, that something along these lines represents the correct analysis of whquestions under the copy theory of movement.

Let us also consider the derivation of the PF corresponding to (25), which involves the PF version of the economy principle in (15). Recall that this principle only allows pronunciation of those copies necessary to (a) recover all the features of the constituents involved, and (b) create a morpho-phonologically wellformed string. Now, since the decision of which copy to pronounce or interpret is done locally at $\mathrm{PF}$ and LF respectively, there is no reason for the copies privileged at PF to match those privileged at LF. In the particular case of English, the whole lower copy is deleted (however one wishes to encode this fact), resulting in the structure in (28). 
(28) Phonetic Form for (25)

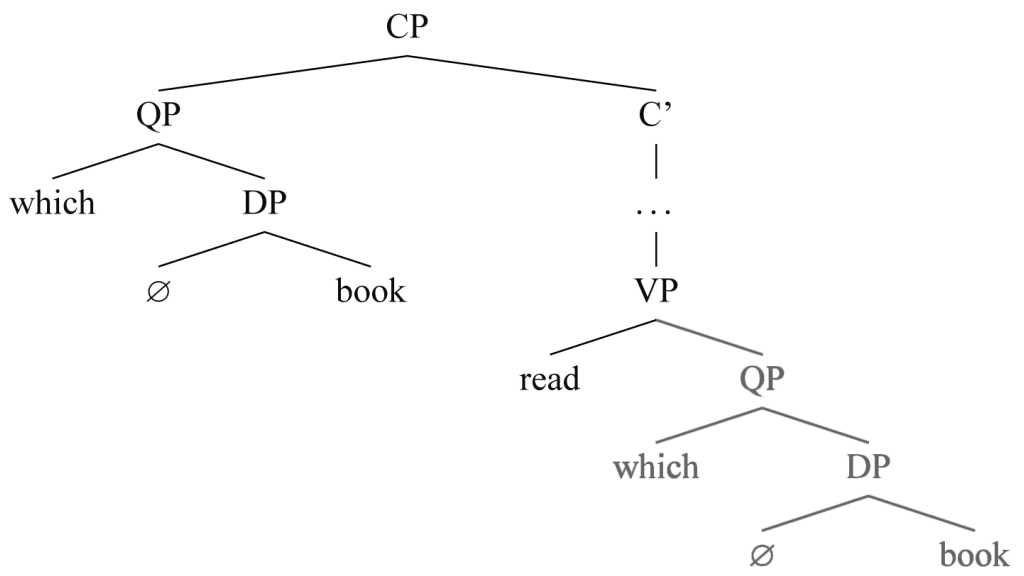

This concludes the discussion of the copy theory of movement. We have seen that, by combining Johnson's decompositional analysis of quantifiers with the copy deletion mechanism in (16), we are able to derive a well-formed LF and PF for wh- questions in a wh- fronting language. The next section will aim at reproducing the same results under a multidominance theory of movement.

\section{A multidominance implementation and its consequences}

The copy-theoretic approach developed above is based on deleting certain copies of constituents. This mechanism cannot be directly applied to a multidominance structure. The reason is that, under multidominance, movement involves one single constituent, immediately dominated by more than one mother node. Therefore, any modifications to said constituent will carry over to every position in the structure it is linked to. This is clearly too strong an analysis, as LF deletion of this constituent would eliminate it altogether, beyond L-recoverability. There is, however, an alternative. The multidominance equivalent to copy-theoretic movement chains are the branches that connect a unique constituent to various structural positions. These branches are the objects that should be affected by LF deletion (and not the constituent itself), as they are the analogues to multiple copies. This means that, given example (25) -repeated as (29)-, the multidominance syntactic structure in (30) translates into the LF in (31). Again, I use a light gray shade to represent the branch deleted at LF.

(29) Which book did Randy buy? 
(30) A multidominance syntax for (30)

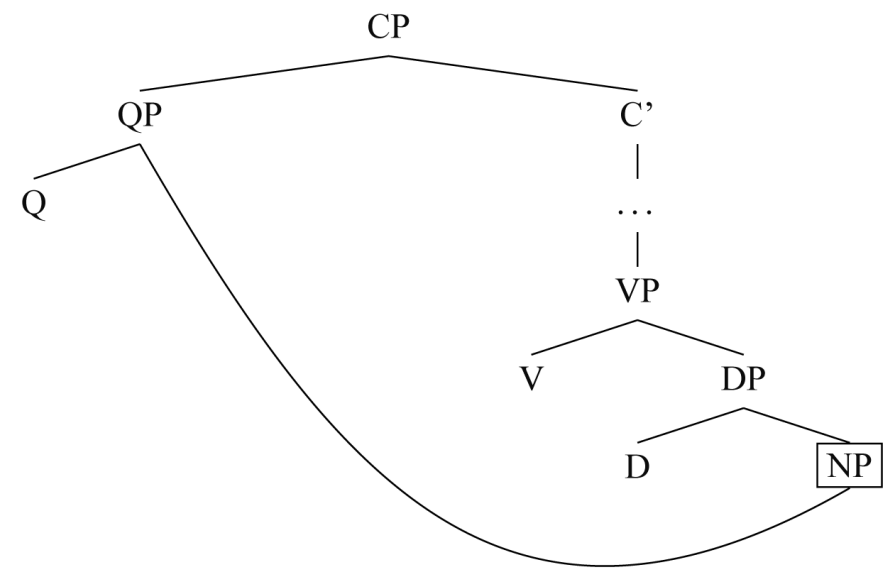

(31) Logical Form for (30)

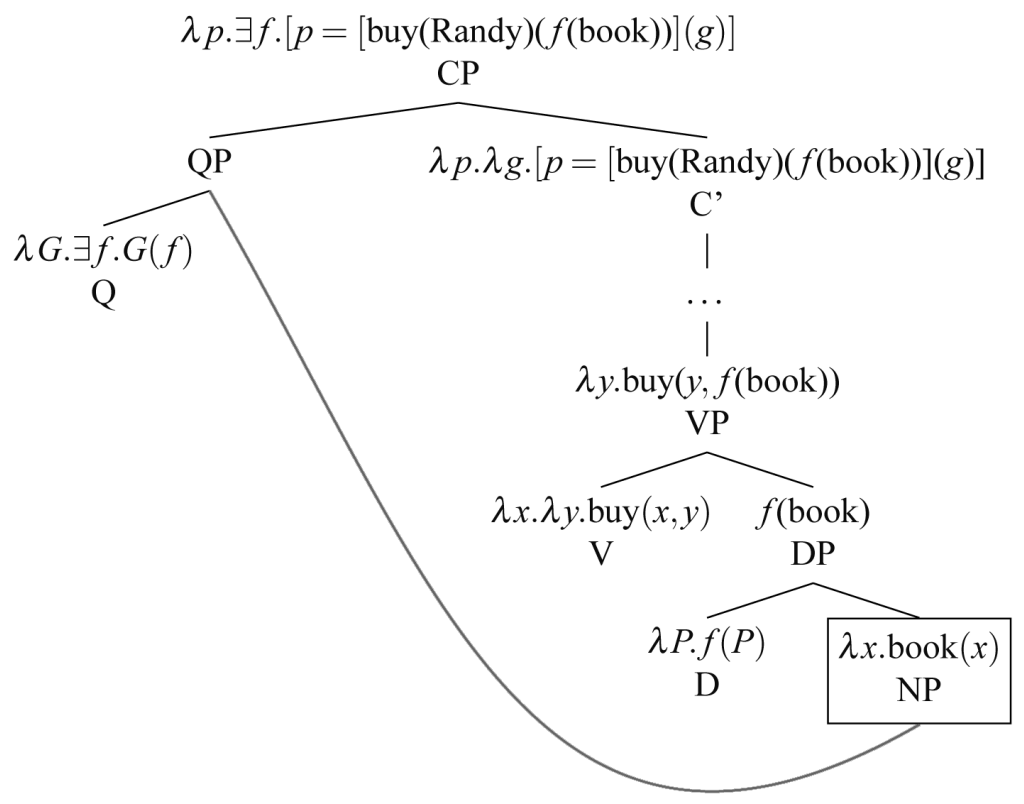

Similarly, the correct pronunciation is derived by letting PF delete the branch that connects NP to D, while preserving the one that connects NP to Q. This results in pronunciation of NP at the scope position, as corresponds to English. Note that 
this analysis requires the assumption that $\mathrm{Q}$ is spelled out at PF as a wh- item, while D is silent. ${ }^{7}$

(32) A multidominance PF for (30)

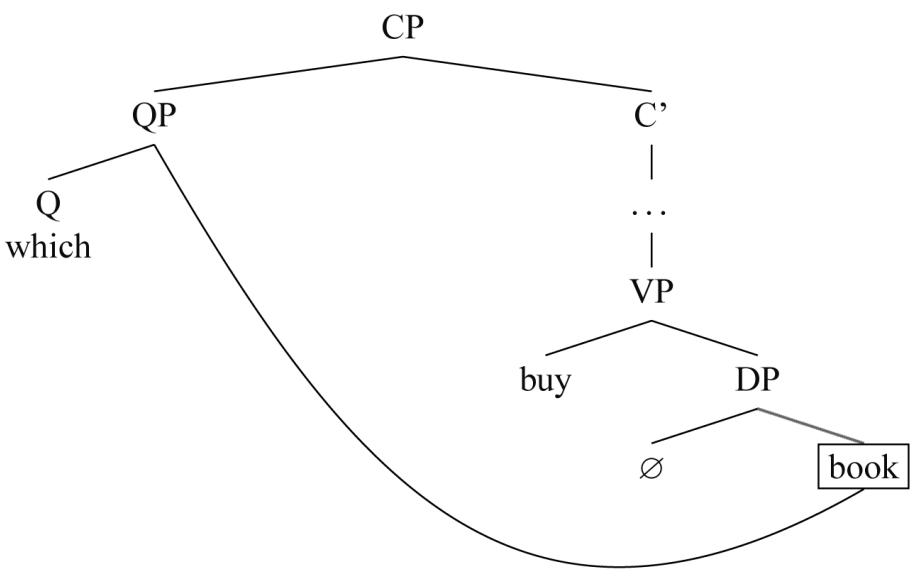

There is, nonetheless, a non-trivial question that arises with respect to these trees. In (30), I have followed Johnson (2007) in merging Q directly in the scope position, and then linking it to NP. This linkage is necessary to account for the fact that wh- chains are sensitive to island boundaries -at least inasmuch as we want to take this type of multidominance branches to be the equivalent to copy-theoretic movement chains. However, if we assume that multidominance branches are equivalent to movement operations under the copy theory of movement, then (30) raises the same problem as (24) in section 4 -i.e., we are performing an illegal operation of "movement" into a complement position. Consequently, we reach the same conclusion as we did in section 4 (see also Cable 2007): in English and other wh- fronting languages, $\mathrm{Q}$ must be merged in the argument position, and then raise to its scope position pied-piping its DP complement. ${ }^{8}$

7. Johnson (2007) argues that D being silent is an idiosyncracy of English: other languages do pronounce $\mathrm{D}$, and in these languages, it surfaces as a resumptive pronoun.

8. It is important to bear in mind that this issue arises because we are considering a wh- fronting language. In order to capture the wh- fronting effect, the complement of Q must be represented in the scope position, so as to provide the right input to $\mathrm{PF}$, and this is what forces us to posit a movement/multidomination operation. Note, however, that this issue does not arise in wh- in situ languages, where there is no need to represent the complement of $Q$ in the scope position. In this class of languages, it ought to be possible to merge $\mathrm{Q}$ directly in the scope position, without linking it to the argument position at all, and still get correct semantics. Hagstrom (1998:ch. 2) shows that this is indeed an option in Japanese and Sinhala. Even more interestingly, he also shows that this nonmovement strategy co-exists with a strategy in which $\mathrm{Q}$ is merged in the argument position and then moves. Studying the implications of wh- in situ languages for the analysis proposed here is a fascinating topic, but for reasons of space, I must defer it to future research. 
Let us assume, then, that both Q is merged in the argument position, and then linked to the scope position. While this alternative does not suffer of the problem just discussed, its proper implementation has to be thought through carefully. Consider, to begin with, a situation in which it is the whole QP that is remerged to the scope position, as in the following syntactic structure (in this particular case, remerger happens on a right branch purely for typographical convenience).

\section{An alternative syntax: remerger of $Q P$}

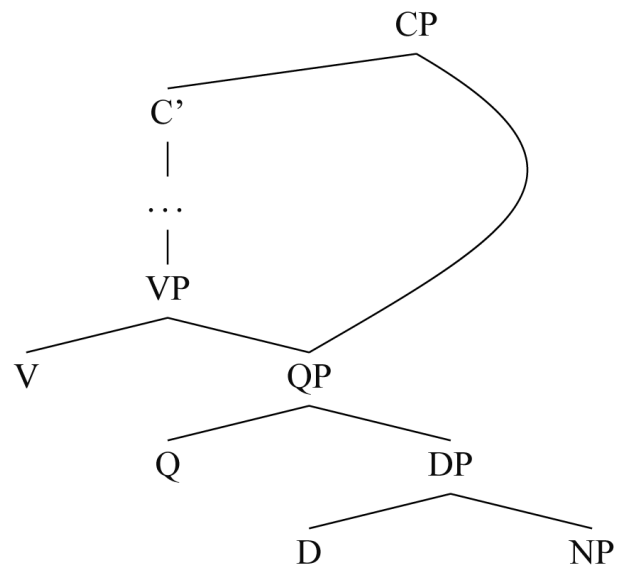

This structure is problematic. While the wh- fronting order of English could be easily derived by deletion of the branch connecting VP and QP at PF (so that the whole QP is pronounced at the scope position), it is simply not possible to derive a well-formed LF out of (33). The problem lies on the assumption that, for multidominance, LF deletes branches connecting different structural points. A consequence of this assumption is that multidominance structures do not allow for the type of selective deletion illustrated in (27) above: a multidominated constituent must appear as a whole in either one or the other position, but cannot be split between both positions. Thus, if LF deletes the branch connecting VP and QP, the result is that the whole QP will be interpreted in the scope position. On top of creating compositionality issues, this LF would fail to capture the obligatory reconstruction effect of wh- movement (34). 
(34) An illicit LF: whole QP interpreted high

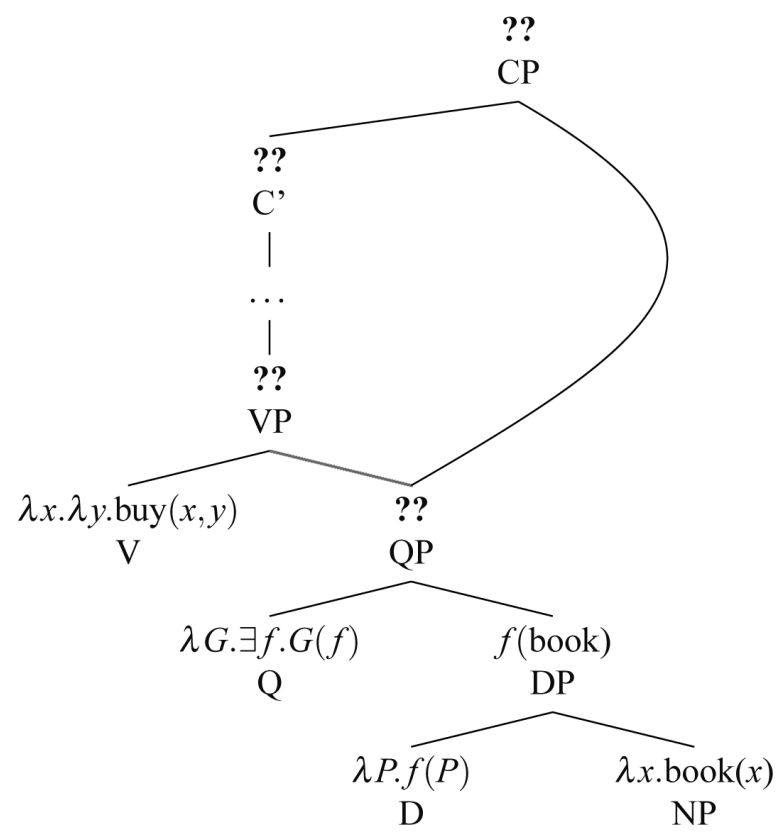

On the other hand, if it is the branch connecting CP and QP that is elided (and again independently of compositionality issues), the whole QP will be interpreted in the argument position, and Q won't be able to take the required scope (35). In either case, we are left with an unusable LF. 
(35) An illicit LF: whole QP interpreted low

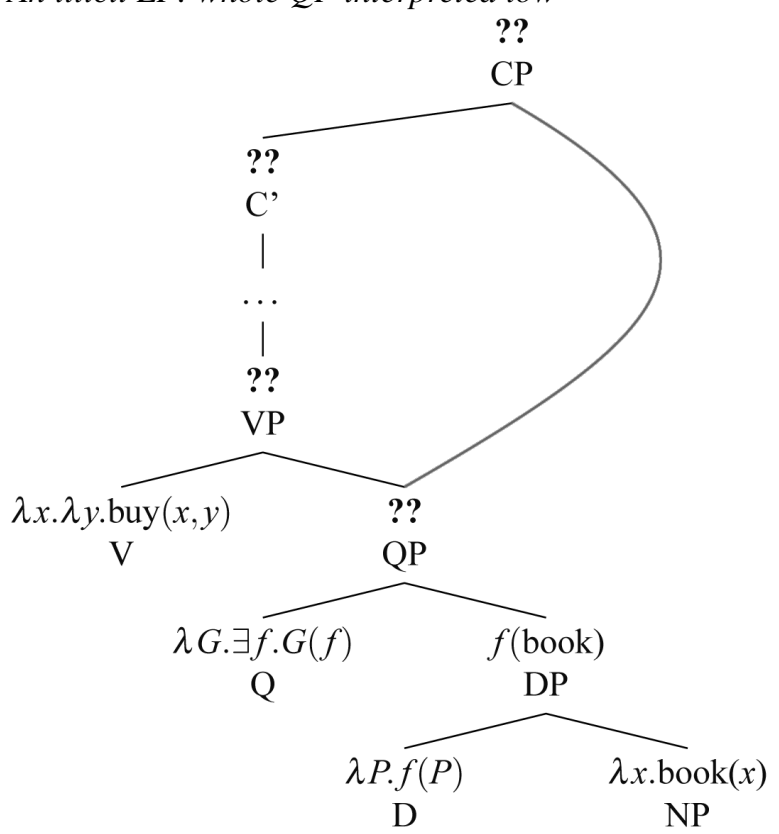

What these representations have taught us is that, beyond the fact that Q needs to be merged low, there is an additional restriction on multidominance structures: remerger of Q to the scope position cannot target the whole QP. As illustrated below, the only way to derive a legitimate $\mathrm{LF}$ is by remerging $\mathrm{Q}$ alone, and then deleting the branch that connects Q to DP (here I am assuming a left-headed VP again purely for typographical reasons). 
(36) An alternative syntax: remerger of $Q$ alone

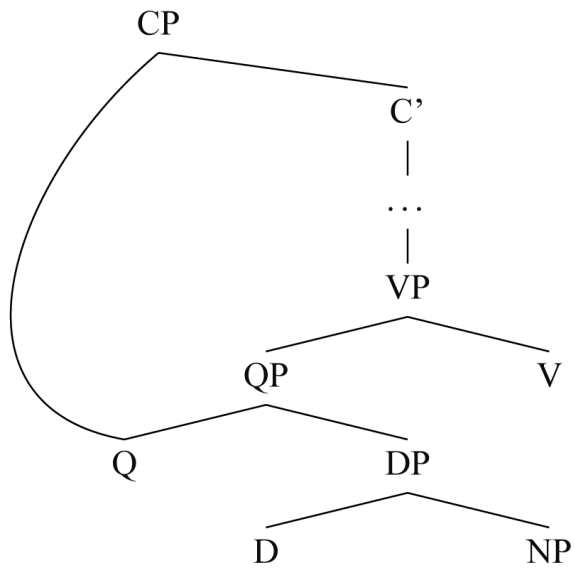

(37) A well-formed multidominance $L F$

$\lambda p . \exists f .[p=[\operatorname{buy}(\operatorname{Randy})(f($ book $))](g)]$

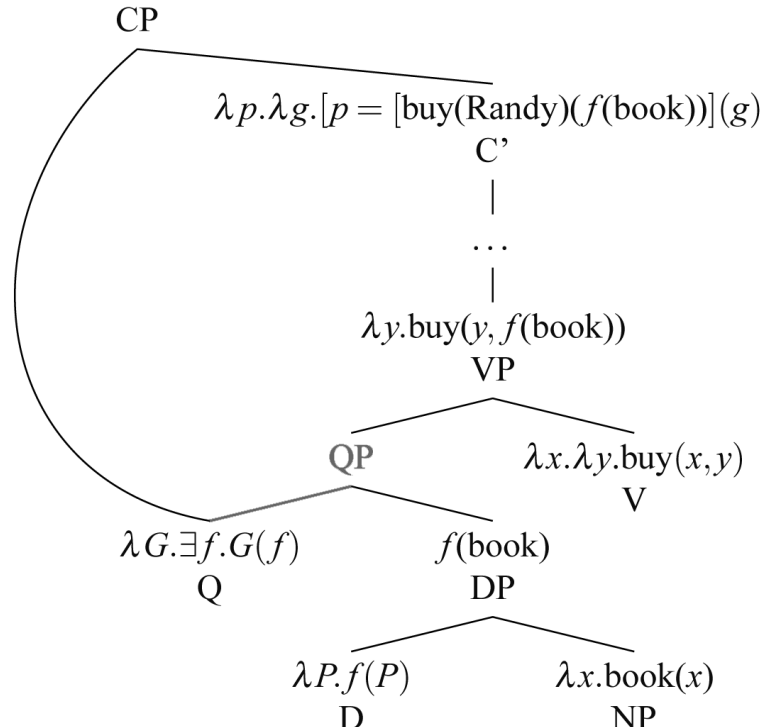

Although by remerging only $\mathrm{Q}$ in the scope position we can derive the correct LF (37), deriving the correct PF is slighty trickier. At PF, what we want is to have the entire DP connected to the scope position, and disconnected from the argument position. The only way to achieve this is by letting PF delete the branch that connects VP to QP, as in (38). 


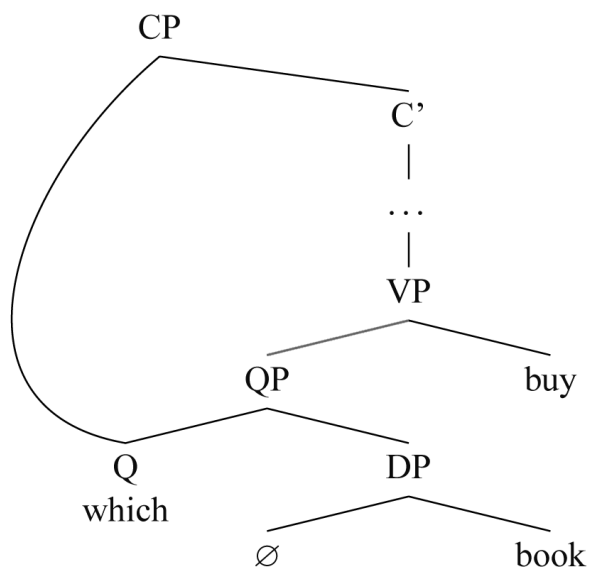

However, as we can see, removing this particular branch leaves us with a very peculiar structure -namely, one in which more than one node (CP and QP) is undominated. Although such structures have been explored occasionally in the literature (cf. Guimarães 2004, Svenonius 2007, and references), their linearization process is far from obvious -see the works just cited for discussion. Suffice it to say that, while the linearization of multiply-rooted structures is technically possible, additional mechanisms are needed beyond what is necessary for the single-rooted structures discussed in section 4 . However, the fact that multiply rooted trees have also been proposed for other phenomena (cf. the works just cited) suggests that the analysis described here is less ad hoc than it might otherwise appear.

\section{General conclusions and outlook}

This article has examined two specific aspects of A-bar chains -an alternative to Trace Conversion and an analysis of reconstruction effects-, in an attempt to figure out how two reasonably plausible accounts of these aspects interact. The purpose of the exercise has been to be able to compare how the copy and multidominance theories of movement perform in this narrow area of grammar. The ultimate goal is to find out some tangible empirical difference between these two approaches to movement, which could then be used to favour one of the approaches over the other.

We have achieved a null result, in that no such differences have been uncovered. Nonetheless, we have also derived an interesting theoretical result-namely, the realization that these two approaches require slightly different assumptions about the mapping from syntax to LF and PF. Starting with the latter, we have seen that a multidominance approach only allows the $\mathrm{Q}$ head to be remerged to the scope position, as remerger of the whole QP leads to an interpretable structure. This makes the syntax of questions look very parallel to their semantics, on the con- 
jecture (Cable 2007) that, at LF, only the $Q$ head can be interpreted at the scope position. On the other hand, under the copy theory of movement, both the Q head and the whole QP can in principle be moved to the scope position (cf. Cable 2007 for an exploration of both options). The question is whether we can get some empirical mileage out of this purely theoretical distinction. In this respect, note that the peculiarity of the semantics of questions discussed in section 2.1 is that Q was defined as a quantifier that takes a single argument (i.e., a set of unsaturated propositions). It is not clear whether the account developed in section 5 can be extended to binary quantifiers (every, some...) and if so, how this extension compares to a copy theoretic alternative. This will remain a topic for future research.

On the PF side, the most notable difference is that multidominance theory allows the creation of multiply-rooted trees, whereas this is not an immediate possibility in the copy theory of movement. Thus, whereas the copy theory of movement can make use of any of the familiar linearization algorithms, a multidominance alternative needs an algorithm that can traverse multiply rooted trees. While such a linearization process is non-standard, it has been proposed independently to account for other phenomena, such as syntactic amalgams (Guimarães 2004) and discontinuous idioms (Svenonius 2007). If Guimarães' and Svenonius’ proposals are on the right track, then the generation of multiply rooted structures cannot be considered a problem for multidomination approaches to movement.

\section{References}

Baker, Carol L. (1970). "Notes on the description of English questions: the role of an abstract question morpheme". Foundations of Language 6:197-219.

Bobaljik, Jonathan (2002). "A-chains at the PF interface: copies and 'covert' movement". Natural Language and Linguistic Theory 20: 197-267.

Cable, Seth (2007). The grammar of $Q$. Doctoral dissertation, MIT.

Corver, Norbert, and Jairo Nunes (2007). The copy theory of movement. Amsterdam: John Benjamins.

Etxeberria, Urtzi (2005). Quantification and domain restriction in Basque. Doctoral dissertation, University of the Basque Country.

Fanselow, Gisbert, and Damir Cavar (2002). "Distributed deletion". In Alexiadou (ed.), Theoretical approaches to universals. Amsterdam: John Benjamins, pp. 65-107.

Fitzpatrick, Justin, and Erich Groat (2005). "The timing of syntactic operations: phases, c-command, remerger, and Lebeaux effects". Handout, ECO'05.

Fox, Danny (2002) "Antecedent contained deletion and the copy theory of movement". Linguistic Inquiry 33:63-96.

Fox, Danny (2003). “On Logical Form”. In Hendrick (ed.) Minimalist syntax. Oxford: Blackwell, pp. 83-123.

Frampton, John (2004). "Copies, traces, occurrences, and all that: evidence from Bulgarian wh- movement". Ms., Northwestern University.

Giannakidou, Anastasia (2004). "Domain restriction and the arguments of quantificational determiners". In Proceedings of SALT 14. Ithaca, NY: CLC Publications, Cornell University, pp. 110-128. 
Guimarães, Maximiliano (2004). Derivation and representation of syntactic amalgams. Doctoral dissertation, University of Maryland, College Park.

Hagstrom, Paul (1998). Decomposing questions. Doctoral dissertation, MIT.

Hulsey, Sarah, and Shoichi Takahashi (2009) "Wholesale late merger: beyond the A/Abar distinction". Linguistic Inquiry 40: 387-426.

Johnson, Kyle (2007). "Determiners and movement". Ms., University of Massachusetts, Amherst.

Kracht, Marcus (2001). "Syntax in chains". Linguistics \& Philosophy 24:467-529. Landau, Idan (2006). "Chain resolution in Hebrew V(P) fronting”. Syntax 9:32-66. Matthewson, Lisa (2001). "Quantification and the nature of cross-linguistic variation". Natural Language Semantics 12:63-127.

May, Robert (1977). The grammar of quantification. Doctoral dissertation, MIT.

Nunes, Jairo (2004). Linearization of chains and sideward movement. Cambridge, Massachusetts: MIT Press.

Reinhart, Tanya (1997). "Quantifier scope: how labor is divided between QR and choice functions”. Linguistics \& Philosophy 20:335-397.

Sauerland, Uli (1998). The meaning of chains. Doctoral dissertation, MIT.

Sauerland, Uli (2004). "The interpretation of traces". Natural Language Semantics 12:63-127.

Sauerland, Uli (2007). “Copying vs. structure sharing: a semantic argument” In Linguistic Variation Yearbook 7, ed. van Craenenbroeck, 27-51. Amsterdam: John Benjamins.

Starke, Michal (2001). Move dissolves into merge: a theory of locality. Doctoral dissertation, University of Geneva.

Svenonius, Peter (2007). "Extending the extension condition to discontinuous idioms". In Pica and Rooryck (ed.). Linguistic Variation Yearbook 5. Amsterdam: John Benjamins, pp. 227-263.

Takahashi, Shoichi (2006). Decompositionality and identity. Doctoral dissertation, MIT. Toyoshima, Takashi (2001). Head-to-spec movement and dynamic economy. Doctoral dissertation, Cornell University.

Vicente, Luis (2007). The syntax of heads and phrases: a study of verb (phrase) fronting. Doctoral dissertation, Leiden University.

Vicente, Luis (2009). "An alternative to remnant movement for partial predicate fronting". Syntax 12: 158-191. 